\title{
A further British case of growth hormone induced Creutzfeldt-Jakob disease
}

\author{
C J Ellis, H Katifi, R O Weller
}

\begin{abstract}
Transmission of Creutzfeldt-Jakob disease (CJD) from cadaveric growth hormone injections had previously been reported in 7 cases, including one from Britain. As a result, the treatment was abandoned in 1985 and superceded by safer recombinant DNA growth hormone injections. Recent reports now record the number of cases worldwide as 23 , but with the incubation period being measured in years, new cases of CJD can still present. We give a detailed report of one of the recent cluster of British cases and aim to highlight the problem to clinicians who may encounter further patients with cadaveric growth hormone induced CJD.
\end{abstract}

$(\Im$ Neurol Neurosurg Psychiatry 1992;55:1200-1202)

Iatrogenic transmission of CJD from cadaveric growth hormone had been reported in 7 patients, including one British case ${ }^{1}$ and it had been hoped that the epidemic was over. ${ }^{2}$ The earlier Raben and Wilhelmi preparation methods for growth hormone had been discontinued $^{3}$ and replaced by highly purified forms ${ }^{4}$ thought unlikely to contain the infective agent. ${ }^{5}$ In turn, these preparations were also withdrawn and replaced by recombinant DNA produced growth hormone; Britain being the first country to grant a product license in October $1985 .^{6}$ Reports have now shown a recent increase in the number of new cases, ${ }^{7-10}$ the worldwide total now being $23 .^{1112}$ The British cohort ${ }^{7}$ of 1908 patients treated with cadaveric growth hormone briefly recorded 6 who had died from CJD including 4 recent cases from 1990. We describe 1 of those 4 recent patients who received all 3 generations of growth hormone over 11 years and then developed the rapidly fatal iatrogenic form of CJD. ${ }^{12}$

\section{Case history}

A 20 year old man had first presented when aged two with a large, cystic craniopharyngioma. This was removed surgically at the Wessex Neurological Centre in 1972 and in 1974, following a local recurrence of the tumour. He made a good recovery although registered blind as the right optic nerve had been severed and the left optic nerve badly damaged. Pituitary replacement therapy was required. Cortisone and thyroxine were given long-term, growth hormone from the age of six to 17 and testosterone from the age of 14 until his presentation aged 20 .

The growth hormone replacement was coordinated from the Royal Hospital for Sick Children in Edinburgh, the city where he attended school. In July 1976 he began to receive 3 injections per week prepared by the Haptree-modified Wilhelmi method (HWP) ${ }^{14}$ and by 1982 he was having the Lowry TPL preparation. ${ }^{4}$ Despite extensive searches the records are incomplete; the batches which he is known to have received include HWP 41,48 , 49, 50 and TPL 22. He did not receive any injections of the Raben preparation. ${ }^{15}$ In May 1985 aged 15, all cadaveric growth hormone injections were discontinued due to the reported cases of CJD transmission. ${ }^{6}$ In December 1985 , aged 16 , he began to receive recombinant DNA produced growth hormone, continuing for a further year when his growth hormone therapy was stopped.

He presented in February 1990 with a 6 month history of vague personality changes when he was noted to be somewhat surly and less efficient at his job as a bank clerk. There had then been a rapid decline with a 3 month history of progressive gait ataxia, limb incoordination and slurred speech. Following presentation he developed spontaneous limb myoclonus, became withdrawn, bedbound and then doubly incontinent over the 2 weeks before admission to hospital.

At that stage his general examination was normal. In the neurological examination spontaneous myoclonic jerks were seen in all 4 limbs and he had a marked startle response to loud noises. Bilaterally there were hand and foot primitive grasp reflexes. He was withdrawn but initially orientated in time and place; his speech demonstrated cerebellar dysarthria. In the cranial nerves, he was blind in the right eye, the left could only perceive light. $\mathrm{He}$ had bilateral optic atrophy with pupils of equal size. The left eye reacted directly and the right eye consensually to light; accommodation could not be determined. The rest of the cranial nerves were intact. In his limbs there was normal tone and power. There were brisk left arm reflexes but reflexes were normal in the right arm and legs, with flexor plantar responses. There was severe cerebellar ataxia in his limbs and trunk; he could not walk or sit up in bed. The sensory system was normal except for mild bilateral proprioceptive loss in his feet. 
Investigations

Blood tests showed normal urea and electrolytes, liver function, calcium, glucose and full blood count with an erythrocyte sedimentation rate of $4 \mathrm{~mm} /$ hour. Viral titres and syphilis serology were negative. A CT brain scan showed evidence of his previous right frontal craniotomy, without hydrocephalus or evidence of brain atrophy. Lumbar puncture revealed clear fluid at normal pressure with less than 1 white cell, no organisms, a normal glucose $(3.5 \mathrm{mmol} / \mathrm{L})$ and protein $(300 \mathrm{mg} / \mathrm{l})$ and negative viral titres. An EEG showed a dominant bilateral slow wave, compatible with degeneration but without the classic spike and wave activity of $\mathrm{CJD}{ }^{16}$

A clinical diagnosis of CJD was made. The patient rapidly declined, becoming mute and requiring total nursing care. He died in late March 1990, and with appropriate precautions ${ }^{17}$ a post mortem examination confirming the diagnosis was carried out locally with further analysis at Southampton General Hospital.

\section{Pathology}

The brain after fixation weighed 1215 grams. There was a defect in the right frontal lobe at the site of removal of the craniopharyngioma. The optic nerves and chaism were atrophic. No cerebral or cerebellar atrophy was noted on external inspection but coronal slices of the cerebral hemispheres revealed mild atrophy on the medial aspects of both frontal lobes; sagittal sections of the cerebellum also revealed mild cortical atrophy.

A whole coronal slice of the cerebral hemispheres, through the level of the amigdala, and selected areas of cerebrum, cerebellum and brain stem were embedded in paraffin and stained with haematoxylin and eosin, Kluver Barrera, Holzer techniques, and by immunocytochemistry for glial fibrillary acidic protein and neurofilament protein. Moderate spongiform change, accompanied by moderate reactive astrocytosis, was seen in the medial and lateral aspects of the frontal lobes. The changes were similar to those seen in a previous case of growth hormone associated Creutzfeldt-Jakob disease. ${ }^{17}$ The temporal cortex showed little spongiform change and no gliosis; the hippocampus was also spared. The most severe spongiform change, and astrocyte proliferation and hypertrophy in the cerebral hemispheres was seen in the thalamus, putamen and globus pallidus. The insula cortex also showed spongiform change and mild astrocytosis. Although spongiform change was only mild in the cortex of the lateral aspect of the occipital lobes, the medial aspects showed marked spongiform change but neither occipital area showed reactive astrocytosis.

Despite the macroscopically normal appearance of the cerebellum, marked spongiform change was seen in the molecular layer of the cerebellar cortex in the lateral hemispheres and in the vermis. This was accompanied by mild Bergmann astrocyte hypertrophy and proliferation. Some loss of Purkinje cells was seen from the vermis but there was little depletion of internal granule cells. The brain stem showed no obvious histological abnormalities.

\section{Discussion}

Creutzfeldt-Jakob disease is a rare progressive neurological disorder characterised by dementia, ataxia and myoclonic movements, and is eventually fatal. ${ }^{19}$ The disease has a prevalence of approximately 1 per million of the population per year, with an average age of death of $57 .^{\circ} \mathrm{CJD}$ is one of a group of transmissible degenerative encephalopathies, from unconventional infective agents, which afflict humans and animals causing diseases which include kuru in humans, scrapie in sheep and bovine spongiform encephalopathy in cows. Pathologically, CJD exhibits a characteristic histological appearance of spongiform change in the neutropil of the cerebral cortex. ${ }^{18}$ The transmissible nature of CJD became clear in 1968 when cerebral biopsy tissue from a patient with the disease was injected into the brain of a chimpanzee which subsequently developed the condition. Iatrogenic transmission of CJD has been reported to occur in a variety of ways. These include cadaveric pituitary-sourced growth and gonadotrophin hormone injections, coroneal transplantation, intracerebral electrode use, cadaveric dura mater graft use and other neurosurgical procedures. ${ }^{1119}$

In 1959 the first clinical use of human growth hormone was described and there were 1908 people in Britain and approximately 10000 people in the USA who had received growth hormone treatment. ${ }^{713}$ The number of pituitary glands used to produce a batch of growth hormone was up to 20000 , with remnants of one batch sometimes being retained and added to future batches; ${ }^{210}$ thus there was the potential to infect many growth hormone vials from only one infected gland.

The human growth hormone preparations used fall broadly into 2 categories. ${ }^{3}$ Before 1975, treatment was almost entirely with growth hormone prepared by the methods of Raben ${ }^{15}$ or Wilhelmi, which was later modified. ${ }^{14}$ Acetone-dried powdered pituitary tissue was subjected to repeated organic solvent extractions (Raben) or alkali and acid precipitations (Wilhelmi). With these methods, the product contained impurities of a much higher molecular weight than growth hormone. ${ }^{3}$ After 1975, the method of extraction was substantially modified using frozen glands and a more gentle extraction procedure incorporating isoelectric precipitation and 2 chromatographic steps. ${ }^{414}$ These modifications were expected to remove the CJD infective agent from the product. ${ }^{3}$ An extensive study of this advanced "Lowry" purification procedure concluded that there was a very low risk that the final product would contain any infective agent. $^{5}$

In primates, direct intracerebral inoculation of the CJD infective agent causes a greater than $90 \%$ disease spread even with small amounts of the agent, but when a small dose is administered by the subcutaneous or intra- 
muscular routes, transmission is irregular and when it does occur is usually followed by extremely long incubation periods. ${ }^{2}$ It is therefore likely that transmission in hypopituitary patients using low concentrations of the CJD infective agent with a peripheral route will infect only limited numbers. ${ }^{2}$ However, the minimum incubation periods in the alreadyidentified patients have ranged from 8 to 28 years, ${ }^{11}$ suggesting that even more cases may be found. Recent work indicates that these patients may have an increased susceptibility to acquire cadaveric growth hormone induced CJD on the basis of their genotype, ${ }^{19}$ an exciting possibility which may be relevant to other transmissible degenerative encephalopathies.

This is the first detailed report of a case of cadaveric growth hormone induced CJD since December 1986, when it was hoped that the "microepidemic" was over. ${ }^{2}$ Over a period of 11 years our patient received both the "Wilhelmi" and the newer "Lowry" type human growth hormone, before receiving recombinant DNA injections, but it remains unclear as to when he was infected. The previous neurosurgery is very unlikely to be the cause, as previous transmissions by this route had much shorter incubation periods of less than 2 years.

Our report raises the possibility that further cases of this unpleasant condition may still occur.

We thank Dr P Kennedy and Dr H Besterman for permission to report their patient and Drs Spencer and Le Gresley for additional help with the case.

1 Powell-Jackson J, Weller RO, Kennedy P, et al. CreutzfeldtJakob disease after administration of human growth hormone. Lancet 1985;2:244-6.

2 Brown P. The decline and fall of Creutzfeldt-Jakob disease associated with human growth hormone therapy. Neurol 1988;38:1135-7.

3 Preece MA. Creutzfeldt-Jakob disease: Implications for growth hormone deficient children. Neuropath Applied Neurobiol 1986;12:509-15.

4 Jones RL, Benker G, Salocinski PR et al. Large scale preparation of highly purified pyrogen-free human growth hormone for clinical use. F Endocrin 1979;82:77-86.

5 Taylor DM, Dickinson AG, Fraser H, et al. Preparation of Growth hormone free from contamination from unconventional viruses. Lancet 1985;2:260-2.

6 Milner RDG. Growth Hormone 1985. BMF 1985;291: 1593-4.

7 Buchanan CR, Preece MA, Milner RDG. Mortality, neoplasia and Creutzfeldt-Jakob disease in patients treated with human pituitary growth hormone in the United Kingdom. BMf 1991;302:824-8.

8 De Villemeur TB, Beauvais P, Gourmelen M, et al. Creutzfeldt-Jakob disease in children treated with growth hormone. Lancet 1991;337:864-5.

9 Macario ME, Vaismon M, Beuscu A, et al. Pituitary growth hormone and Creutzfeldt-Jakob disease. BMF 1991;302: 1149

10 Fradkin JE, Schonberger LB, Mills JL, et al. CreutzfeldtJakob disease in pituitary growth hormone recipients in the United States. $¥ A M A$ 1991;265:880-4.

11 Preece MA. Creutzfeldt-Jakob disease following treatment with human pituitary hormones. Clin Endocrinol 1991;34: 527-9.

12 Brown P, Preece MA, Will RG. 'Friendly fire' in medicine: hormones, homografts, and Creutzfeldt-Jakob disease. Lancet 1992;340:24-7.

13 Brown P. Human growth hormone therapy and CreutzfeldtJakob disease: a drama in three acts. Paediatrics 1988; 81:85-92.

14 Mills JB, Ashworth RB, Wilhelmi $\mathrm{AE}$, et al. Improved method for the extraction and purification of human growth hormone. F Clin Endocrinol Metab 1969;29: $1456-9$

15 Raben MS. Human growth hormone. Rec Progr Horome Res 1959;15:71-114

16 Knight R. Creutzfeldt-Jakob disease. $B r f$ Hosp Med 1989;14:165-71.

17 Rosenberg RN, White CL, Brown P, et al. Precautions in handling tissues, fluids and other contaminated materials from patients with documented or suspected Creutzfeldtakob disease. Committee on health care issues ANA Amm Neurol 1986;19:75-7.

18 Weller RO, Steart PV, Powell-Jackson JD. Pathology of Creutzfeldt-Jakob disease associated with pituitaryderived human growth hormone administration. Neuropath Applied Neurobiol 1986;12:117-29.

19 Weller RO. Iatrogenic transmission of Creutzfeldt-Jakob Weller RO. Iatrogenic transmission of
disease. Psychological Med 1989;19:1-4.

20 Collinge J, Palmer MS, Dryden AJ. Genetic predisposition to iatrogenic Creutzfeldt-Jakob disease. Lancet 1991;337: 1441-2. 